\title{
BrachyView, a novel in-body imaging system for HDR prostate brachytherapy: experimental evaluation
}

\author{
M. Safavi-Naeini, Z. Han, S. Alnaghy, D. Cutajar, M. Petasecca, M.L.F. Lerch \\ Centre for Medical Radiation Physics, \\ University of Wollongong, Australia \\ D.R. Franklin \\ Faculty of Engineering and Information Technology, \\ University of Technology, Sydney, Australia \\ J. Bucci \\ St George Hospital Cancer Care Centre, Australia
}

\author{
M. Carrara \\ Fondazione IRCCS Istituto Nazionale dei Tumori, Milan, Italy \\ M. Zaider \\ Memorial Sloan Kettering Cancer Center, U.S.A.
}

A.B. Rosenfeld*

Centre for Medical Radiation Physics,

University of Wollongong, Australia

(Dated: October 19, 2015) 


\begin{abstract}
Purpose: This paper presents initial experimental results from a prototype of HDR BrachyView, a novel in-body source tracking system for HDR brachytherapy based on a multi-pinhole tungsten collimator and a high resolution pixellated silicon detector array. The probe and its associated position estimation algorithms are validated and a comprehensive evaluation of the accuracy of its position estimation capabilities is presented.
\end{abstract}

Method: The HDR brachytherapy source is moved through a sequence of positions in a prostate phantom, for various displacements in $x, y$ and $z$. For each position, multiple image acquisitions are performed, and source positions are reconstructed. Error estimates in each dimension are calculated at each source position and combined to calculate overall positioning errors. Gafchromic film is used to validate the accuracy of source placement within the phantom.

Results: More than $90 \%$ of evaluated source positions were estimated with an error of less than one millimetre, with the worst-case error being $1.3 \mathrm{~mm}$. Experimental results were in close agreement with previously published Monte Carlo simulation results.

Conclusion: The prototype of HDR BrachyView demonstrates a satisfactory level of accuracy in its source position estimation, and additional improvements are achievable with further refinement of HDR BrachyView's image processing algorithms.

PACS numbers: 87.53.Jw,87.55.N-,87.55.Qr,87.56.Fc,87.57.nj,87.57.uq

Keywords: in vivo dosimetry, brachytherapy, HDR, BrachyView 


\section{INTRODUCTION}

High dose rate brachytherapy is currently one of the leading treatment options for clinitween the delivered dose and the desired dose calculated by the treatment planning system $(\mathrm{TPS})^{14}$. Carrara et al. have successfully tested this QA method on six patients undergoing HDR PBT and used it to quantify intra-fraction prostate motion ${ }^{15}$. 
An alternative real-time dose monitoring method for HDR PBT uses dosimeters based

on an $\mathrm{Al}_{2} \mathrm{O}_{3}$ : $\mathrm{C}$ crystal coupled by optical fibre to a photomultiplier or solid state photon detector $^{16-18}$. Such detectors can measure dose both via radioluminescence (yielding an instantaneous dose measurement) or through optically stimulated luminescence (integrated over the exposure time since the last optical readout pulse). The former mode of operation is most appropriate for real-time dosimetric QA in HDR PBT. Cartwright et al. performed in-rectum dosimetry using 16 fibre-coupled scintillators placed in a trans-rectal applicator, and obtained measurements within $3 \%$ of the intended dose as calculated by the TPS. This method is able to provide an estimate of source position in one dimension with an accuracy of approximately $2 \mathrm{~mm}^{19}$. Although fibre-coupled scintillator dosimetry can accurately monitor the dose rate at selected sampling positions in real-time, it provides insufficient information to be able to determine the position of the source, and the spatial distribution of dose cannot be accurately estimated.

Wang et al. proposed a method to validate the source delivery accuracy of the afterloader in one dimension in real-time. Two GaN dosimetric probes were used to track the horizontal displacement of the source within a predefined catheter location. Although sub-millimetre accuracy is achieved, this method is very limited in application since source position information is only available in one dimension (parallel to the catheter) and the position of the catheter relative to the probes has to be known in advance and assumed to be correct ${ }^{20}$. A more sophisticated QA system, capable of providing detailed pretreatment QA information including source position in the phantom volume with sub-millimetre accuracy and source dwell and transit time was proposed and investigated by Espinoza et al. ${ }^{21}$. Using the Magic Phantom, a phantom with two rows of ten uniformly spaced catheters with the Magic Plate silicon diode array placed in-between to track the source position, dwell time as well as the two dimensional dose distribution were determined ${ }^{21,22}$.

Methods using an external imaging device combined with a pinhole or analogous collimator have been proposed and aim to provide intra-fraction real-time source tracking. However, due to the large source to collimator distance, these methods either require a lengthy acquisition time (more than $2 \mathrm{~s}$ for a HDR source of typical activity), which is too slow for real-time source tracking, or suffer from an unacceptable positioning error (larger than $3 \mathrm{~mm}$, suggested by Tiong et al. $\left.{ }^{23}\right)^{24-26}$.

BrachyView is a family of in-body imaging systems developed at the University of Wol- 
longong's Centre for Medical Radiation Physics (CMRP), which combine a multi-pinhole tungsten collimator with an array of Timepix detectors ${ }^{27}$. BrachyView aims to provide reliable intra-fraction realtime source tracking. The application of BrachyView in low dose rate (LDR) PBT (LDR BrachyView) has been investigated using ${ }^{125}$ I seeds and a PMMA phantom. Sub-millimetre accuracy can be obtained within an acquisition time of $3 \mathrm{~s}$ when seeds are placed within $20 \mathrm{~mm}$ of the collimator's upper surface; this error increases with source-to-detector distance and reaches $3 \mathrm{~mm}$ when the seeds are $60 \mathrm{~mm}$ away $^{28}$. The capability of BrachyView (with the collimator removed) to additionally perform X-ray imaging of soft tissue in PBT has also been evaluated ${ }^{29}$. The related HDR BrachyView device, intended for QA of high dose rate (HDR) PBT, has previously been characterised in detail via Monte Carlo simulations, which predicted that a maximum error of $1.5 \mathrm{~mm}$ within the maximum extent of the prostate volume could be obtained within a sub-second acquisition time (with the source up to $45 \mathrm{~mm}$ away from the collimator's top surface) ${ }^{30}$. Furthermore, additional Monte Carlo simulations and preliminary experimental work have demonstrated that the dose enhancement at the rectal wall resulting from backscatter from the $H D R$ BrachyView probe's tungsten shell is negligible provided that the probe is encapsulated in a tissue-equivalent plastic shell with a thickness of at least $0.5 \mathrm{~mm}^{31}$.

The aim of this paper is to experimentally evaluate the efficiency and accuracy of source position tracking using the HDR BrachyView probe. The estimated source position will ultimately be registered to the prostate by combining HDR BrachyView with a transrectal ultrasound (TRUS) probe $^{30}$.

The remainder of this paper is arranged as follows: the experimental configuration and the source position reconstruction algorithm are discussed in Section II; the source position estimates are calculated and compared with planned positions in Section III; a thorough analysis of the results including a discussion of the possible sources of error is presented in Section IV; and finally, conclusions and possible approaches to improve accuracy are given in Section V.

\section{MATERIAL AND METHODS}

A prototype HDR BrachyView probe has been developed at the Centre for Medical Radiation Physics, University of Wollongong and its accuracy in tracking the HDR source for 


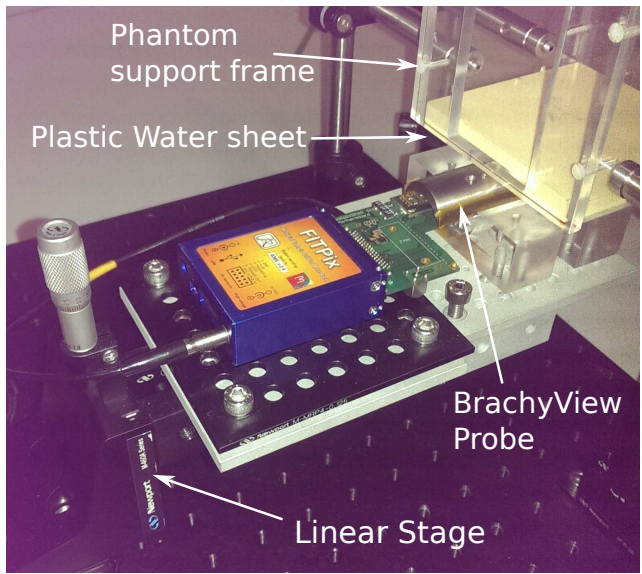

(a)Experimental Configuration

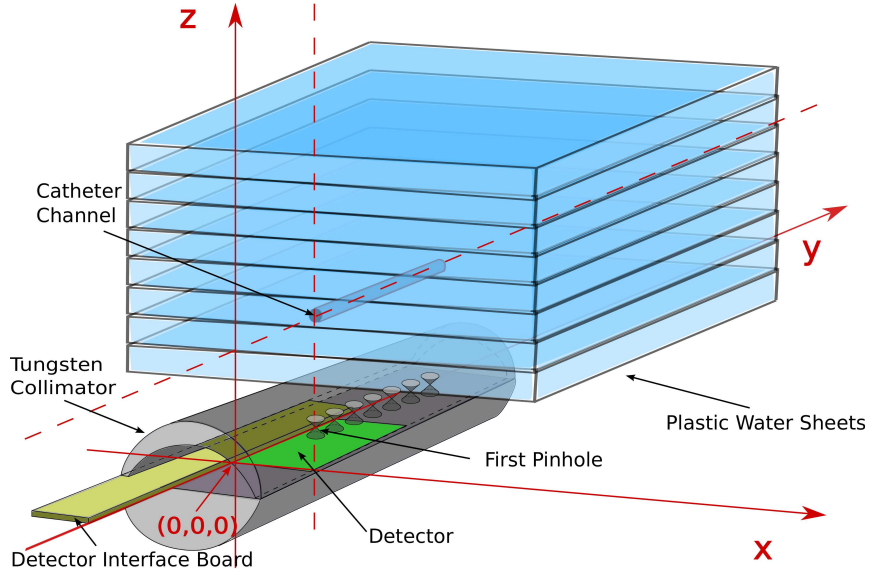

(b)Schematic

FIG. 1. The prototype HDR BrachyViewprobe (actual and schematic with coordinate system), together with its linear translational stage and the phantom.

sub-second dwell times was evaluated experimentally. The design of the prototype and the experimental configuration is described in Section II A. A comprehensive description of the source tracking process is provided in Section II B.

\section{A. Experimental configuration}

The experiment was conducted at the HDR Brachytherapy facilities at the St. George Hospital Cancer Care Centre, Kogarah. The configuration consists of four main components: the HDR BrachyView prototype probe, a linear translational stage, a simplified prostate phantom, and a HDR brachytherapy source and afterloader mechanism.

The experimental configuration is shown in FIG. 1(a) and a schematic of the probe and the Solid water phantom is illustrated in FIG. 1(b).

The prototype probe consists of a semi cylindrical tungsten collimator, coupled to a semicylindrical aluminium base of the same diameter which houses two tiled Timepix detector arrays. The two $14 \mathrm{~mm} \times 14 \mathrm{~mm}$ Timepix detectors provide a $14 \mathrm{~mm} \times 28 \mathrm{~mm}$ sensitive detection area ${ }^{27}$. Together with their associated microprocessor-controlled USB2 interfaced readout system (Fitpix) they give the probe the capability of imaging the source within half of the target volume (FIG. 2(a)), i.e. the prostate phantom, at a frame rate of up to 400 images per second and with very low electronic noise. A global cartesian coordinate system was introduced and is shown in FIG. 1(b), with its origin placed at the top left corner of 


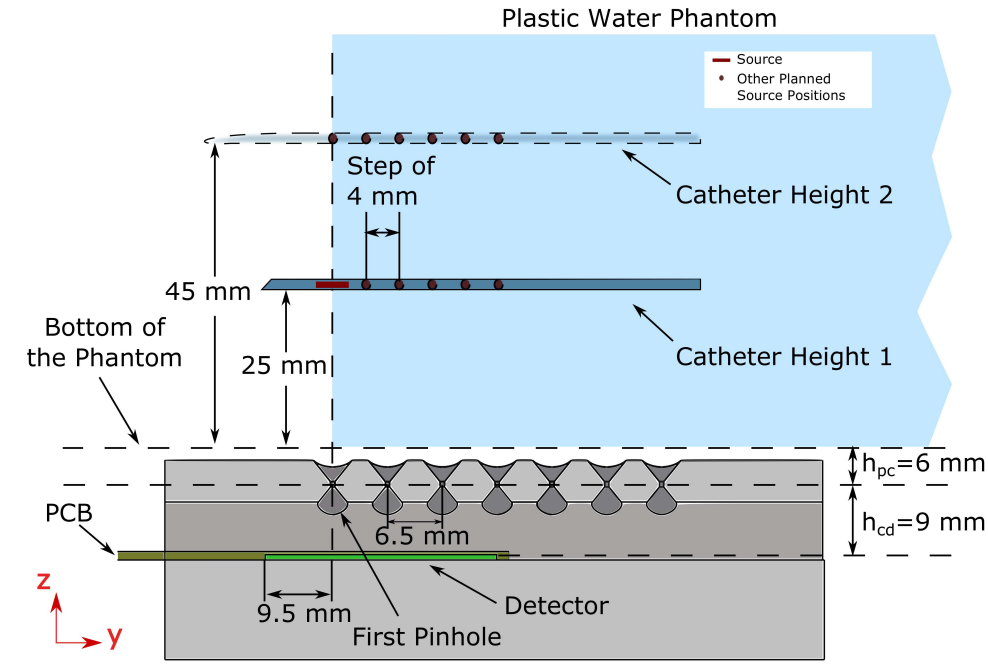

(a)Longitudinal cross-section



(b)Transversal cross-section

FIG. 2. The cross-sections of the HDR BrachyView prototype probe, the phantom and the planned source positions. The catheter positions used in the experiment are marked within the phantom.

the first detector (first row and first column of the imaging plane).

Seven double cone equidistant pinholes (centre-to-centre spacing of $6.5 \pm 0.05 \mathrm{~mm}$ ) were drilled onto the collimator, parallel to the central axis of the detectors (along the $y$ axis) and are shown in FIG. 2(a). The acceptance angle of the double cone pinhole is $90^{\circ} \pm 0.5^{\circ}$. A cylindrical channel with a diameter of $0.5 \mathrm{~mm}$ and a length of $0.5 \mathrm{~mm}$ connects the top and bottom cones.

The geometry of the truncated double cone pinholes and their preferred placement have been discussed in previous publications ${ }^{30,31}$. However, the geometry of the prototype board on which the Timepix chips were mounted was such that it was not possible to align the centre of the detectors with the centre of the collimator tube. Therefore the pinholes were fabricated with the same offset as the detectors, as illustrated in FIG. 2(b).

It was demonstrated previously that four tiled detectors are needed to cover the whole prostate volume ${ }^{30}$. However, the axial symmetry of the phantom allows for the evaluation of the probe performance by moving the source within half of the field of view (FOV).

The phantom comprises a series of $90 \mathrm{~mm} \times 90 \mathrm{~mm}$ Computerized Imaging Reference Systems (CIRS) Plastic Water ${ }^{\circledR}$ LR sheets, each with a thickness of either $5 \mathrm{~mm}$ or $10 \mathrm{~mm}$ and optimised for photon energies from $15 \mathrm{keV}$ to $8 \mathrm{MeV}$. A $2 \mathrm{~mm}$ diameter channel was etched halfway along the central axis ( $y$ axis) of a $90 \mathrm{~mm} \times 90 \mathrm{~mm} \times 10 \mathrm{~mm}$ Plastic Water sheet. 
TABLE I. Planned Source Positions for each test.

\begin{tabular}{cccc}
\hline \hline & \multicolumn{3}{c}{ Source Positions } \\
Test & $\mathrm{x}(\mathrm{mm})$ & $\mathrm{y}(\mathrm{mm})$ & $\mathrm{z}(\mathrm{mm})$ \\
\hline 1 & 7.0 & & 40 \\
2 & 7.0 & & 60 \\
3 & 9.5 & & 60 \\
4 & 9.5 & 5 positions from & 40 \\
5 & 12.0 & 13.5 to 29.5 with & 40 \\
6 & 12.0 & a step of 4 & 60 \\
7 & 14.5 & & 60 \\
8 & 14.5 & & 40 \\
\hline \hline
\end{tabular}

This allowed for the insertion of a catheter into which a Flexitron ${ }^{192}$ Ir HDR brachytherapy source was remotely moved by a Nucletron Flexitron HDR afterloader. The source was moved into five preplanned positions along the $y$ axis, starting at the entrance of the catheter within the phantom and moving in the positive $y$ direction in steps of $4 \mathrm{~mm}$.

The Plastic Water sheets were arranged to place the catheter either $40 \mathrm{~mm}$ or $60 \mathrm{~mm}$ above the detector plane of the HDR BrachyView probe along the $y$ axis, as illustrated in FIG. 1(b) and FIG. 2. The upper catheter position is set at $60 \mathrm{~mm}$, since in clinical practice, $60 \mathrm{~mm}$ is generally considered the upper limit of the possible distance between rectal wall and the most distant needle placed inside the prostate for treatment.

A linear translational stage with a $1 \mu \mathrm{m}$ accuracy was used to move the probe laterally (along the $x$ axis) in four $2.5 \mathrm{~mm}$ steps, relative to the phantom and the catheter (FIG. 2(b)). A total of eight catheter positions were thus simulated. The position of the phantom relative to the probe is shown in FIG. 2(a), where the front face of the phantom is offset from the centre of the first pinhole by $0.3 \mathrm{~mm}$, due to the mechanical constraints of the experimental apparatus.

The source air kerma strength at the time of irradiation was $19.5 \mathrm{mGyh}^{-1} \mathrm{~m}^{2}$, corresponding to approximately $50 \%$ of the full source strength of $40.7 \mathrm{mGyh}^{-1} \mathrm{~m}^{2}$. Source dwell times in each position were calculated as $43 \mathrm{~s}$, equivalent to $20.6 \mathrm{~s}$ for a full strength source. A list of all planned source positions is provided in Table I. 


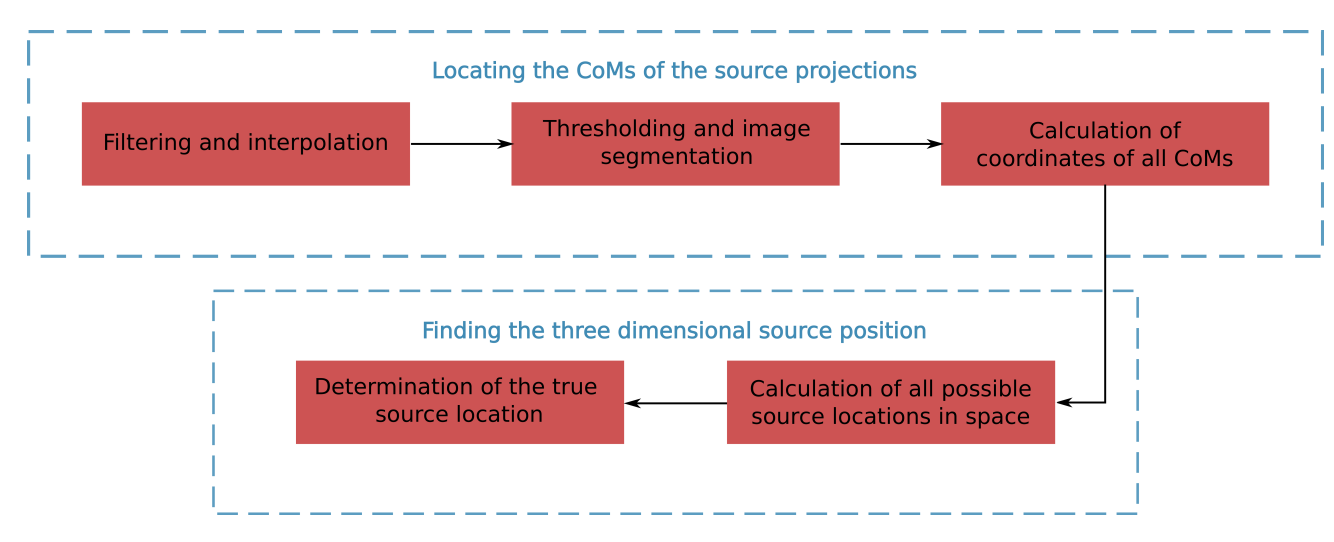

FIG. 3. The process of calculating the source position within the prostate volume.

\section{B. Reconstruction method}

The two Timepix chips are calibrated and equalised to ensure a uniform response across the full array and configured to continuously acquire and record frames with an exposure time of 0.1 second (with a short dead time between each frame acquisition). Groups of ten consecutive frames are combined to produce images with an effective exposure time of one second; an average of 18 such images are collected for each source position, with any frames captured during source movement discarded. The images collected for each source position are then passed through a $3 \times 3$ median filter, after which the small gap between the chips is filled via bilinear interpolation. The projections are separated via a simple image segmentation process and then thresholded. The centres of mass (CoMs) of all visible projections, which are used as an approximation for the projected source centre, can then be computed. The CoMs are then backprojected through their corresponding pinholes and the three dimensional source location, defined as the point with the minimum mean square distance to all of these rays, is found for each one-second acquisition ${ }^{30}$. A unique source location can be found even if the source is not visible through all pinholes; all potential candidate source positions are calculated, and the point with the shortest distance to the $\mathrm{CoM}$ of the brightest projection is taken as the true source position ${ }^{30}$. The procedure is repeated for all 18 images collected at each source position, and the mean and standard deviation of the source position estimate are calculated across the image set for each source position.

A block diagram of the steps involved in the calculation of source location is shown in FIG. 3. 
At every position, the source is seen by multiple pinholes, with varying source to pinhole distance. The photon flux is, to the first approximation, inversely proportional to the square tracked by the HDR BrachyView probe.

\section{RESULTS}

The mean estimated source positions are plotted in FIG. 5, both in three dimensions and projected on to the three main planes of the coordinate system (defined in Section II A); 


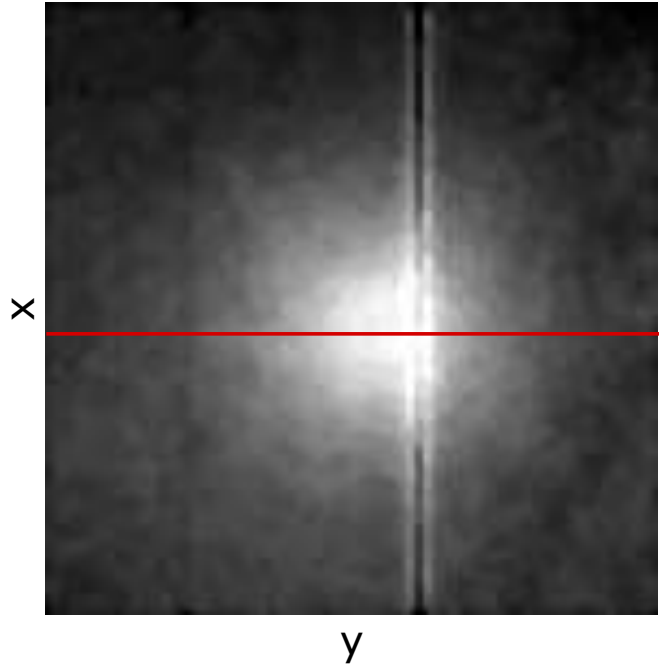

(a)Before interpolation



(c)Line profile, before interpolation

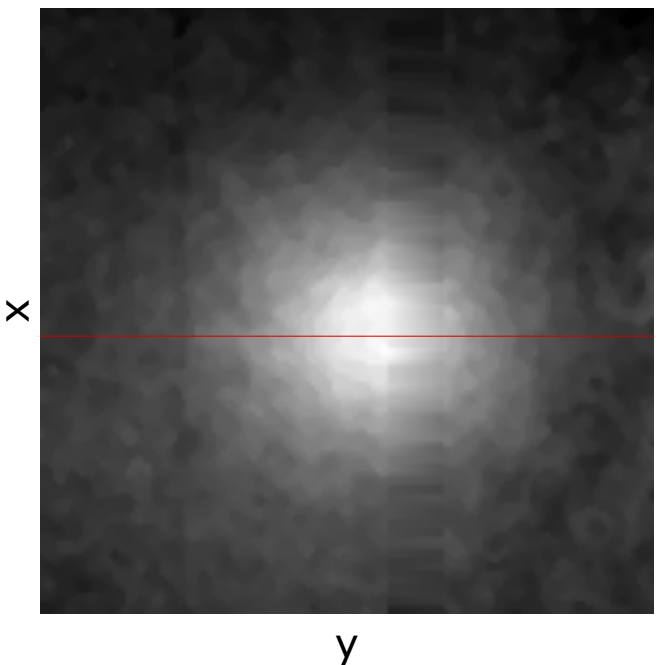

(b)After interpolation

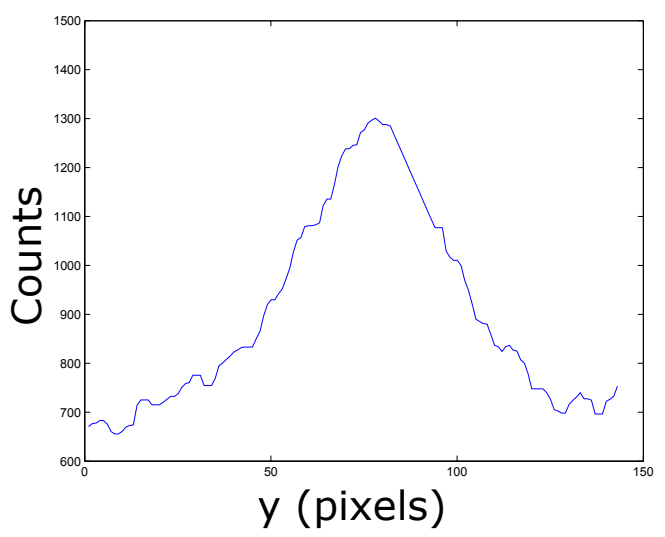

(d)Line profile, after interpolation

FIG. 4. Source projection shown at the boundary of the two Timepix detectors; the non-uniform response of the detector array has been corrected using bilinear interpolation.

The same data together with the standard deviation of the calculated source positions, mean error in each dimension (difference between planned and estimated positions), standard deviation of the mean errors in each dimension for each different catheter (intra-run standard deviation), overall standard deviation of the mean errors in each dimension (interrun standard deviation) and overall mean error are shown in Tables II and III for nominal vertical $(z)$ source-detector distances of $40 \mathrm{~mm}$ and $60 \mathrm{~mm}$ respectively. Larger errors (nominal to measured) and uncertainties (standard deviation between measurements) were found for the larger vertical source-detector distance, especially in the $z$ direction. For $60 \mathrm{~mm}$ nominal vertical source-detector distance, the maximum overall error is $1.3 \mathrm{~mm}$, whereas for 




(a)Three-dimensional view

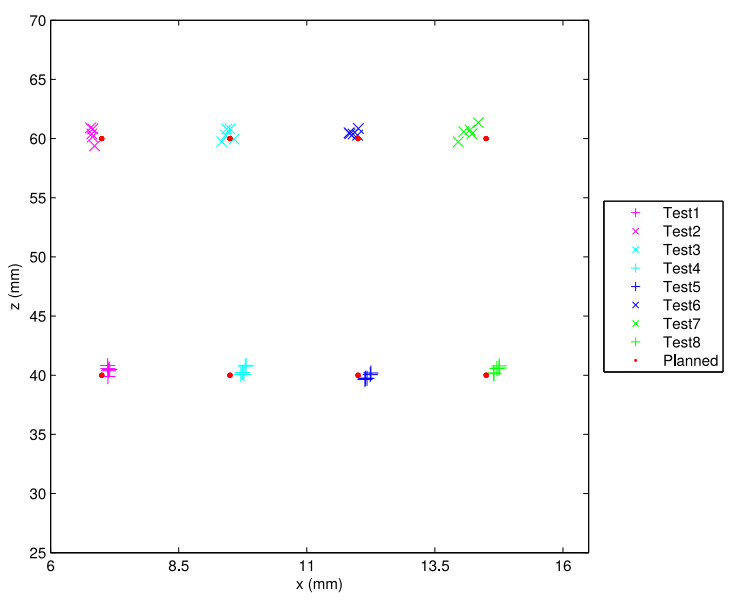

(c)Projection on $x-z$ plane



(b)Projection on $x-y$ plane

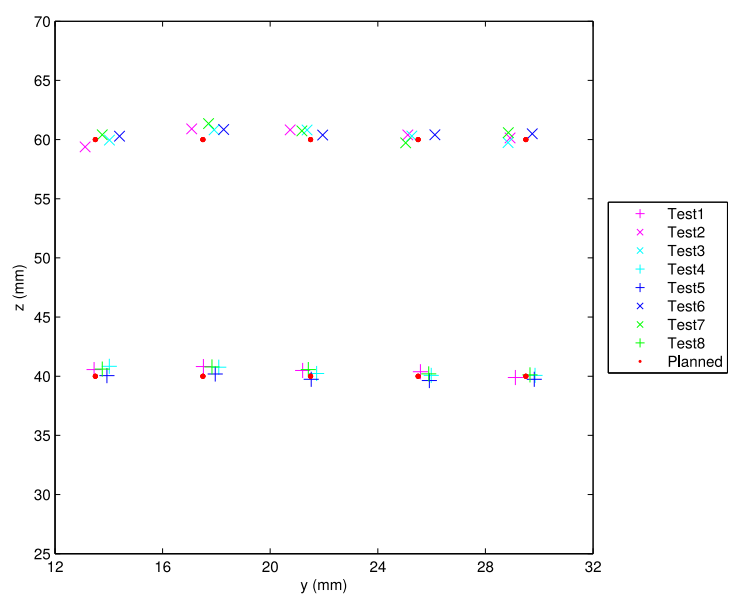

(d)Projection on $y-z$ plane

FIG. 5. Coordinates of calculated and planned source positions, visualised in three dimensions and projected on to the three main planes of the coordinate system.

$40 \mathrm{~mm}$, the maximum overall error is $1 \mathrm{~mm}$. The largest contribution to the overall error in the position estimate for each catheter position (i.e., the intra-run standard deviations) is from the $z$ component. For both source-detector distances, the uncertainties in the $y$ component of the error are always smaller than the overall error values (inter-run standard deviations), while the $x$ component of the position estimate exhibits the smallest intra- and inter-run standard deviations of the three components.

FIG. 6 shows the image recorded on EBT3 Gafchromic film (placed $5 \mathrm{~mm}$ below the source) when the source is located $60 \mathrm{~mm}$ above the top of the detector (the image obtained when the source is located at $z=40 \mathrm{~mm}$ is essentially identical as the source-film distance 
TABLE II. The coordinates of calculated and planned source positions for a vertical source displacement of $40 \mathrm{~mm}$ from the top surface of the detector plane. Standard deviation is denoted $S D$; intra-run means and standard deviations are calculated across 18 one-second $(10 \times 0.1 \mathrm{~s})$ exposures per position. The total error between planned and measured/estimated source position $d_{\text {total }}=\sqrt{d_{x}^{2}+d_{y}^{2}+d_{z}^{2}}$.

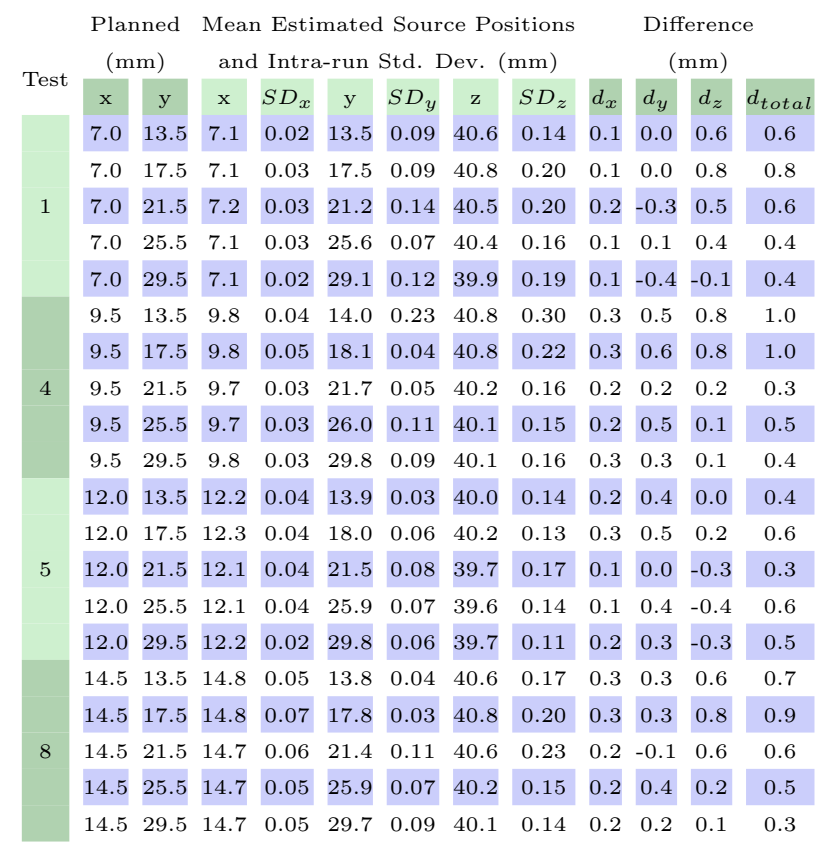

is also $5 \mathrm{~mm}$ ). Three horizontal source displacements were evaluated using this method for each value of $z$ and were used for comparison with the corresponding source positions tracked by the HDR BrachyView probe. Table IV shows the three source positions in the $y$ direction as measured by film and the HDR BrachyView probe, for both values of $z$. The differences between the two methods are up to $0.31 \mathrm{~mm}$ for all measured source positions.

\section{DISCUSSION}

The measurement of the source position when the source was placed at a nominal height of $60 \mathrm{~mm}$ above the detector plane demonstrates the performance of the HDR BrachyView probe in terms of source tracking accuracy and consistency in the worst case for both sensitivity and minification (demagnification). Standard deviations of less than $1 \mathrm{~mm}$ were obtained for all evaluated source positions within a $1 \mathrm{~s}$ acquisition time using a $19.5 \mathrm{mGyh}^{-1} \mathrm{~m}^{2}$ source (equivalent to an acquisition time of $0.5 \mathrm{~s}$ for a full strength source). The increase in stan- 
TABLE III. The coordinates of calculated and planned source positions for a vertical source displacement of $60 \mathrm{~mm}$ from the top surface of the detector plane. Standard deviation is denoted $S D$; intra-run means and standard deviations are calculated across 18 one-second $(10 \times 0.1 \mathrm{~s})$ exposures per position. The total error between planned and measured/estimated source position $d_{\text {total }}=\sqrt{d_{x}^{2}+d_{y}^{2}+d_{z}^{2}}$.

\begin{tabular}{|c|c|c|c|c|c|c|c|c|c|c|c|c|}
\hline \multirow{2}{*}{ Tes } & \multicolumn{2}{|c|}{$\begin{array}{l}\text { Planned } \\
(\mathrm{mm})\end{array}$} & \multicolumn{6}{|c|}{$\begin{array}{l}\text { Mean Estimated Source Positions } \\
\text { and Intra-run Std. Dev. (mm) }\end{array}$} & \multicolumn{4}{|c|}{$\begin{array}{l}\text { Difference } \\
\quad(\mathrm{mm})\end{array}$} \\
\hline & $\mathrm{x}$ & $\mathrm{y}$ & $\mathrm{x}$ & $S D_{x}$ & $\mathrm{y}$ & $S D_{y}$ & $\mathrm{z}$ & $S D_{z}$ & $d_{x}$ & $d_{y}$ & $d_{z}$ & $d_{\text {total }}$ \\
\hline \multirow{5}{*}{2} & 7.0 & 13.5 & 6.9 & 0.09 & 13.1 & 0.07 & 59.4 & 0.63 & -0.1 & -0.4 & -0.6 & 0.7 \\
\hline & 7.0 & 17.5 & 8 & 0.09 & 17.1 & 0.12 & 60.9 & 0.69 & -0.2 & -0.4 & 0.9 & .0 \\
\hline & 7.0 & 21.5 & 6.8 & 0.07 & 20.7 & 0.08 & 60.8 & 0.63 & -0.2 & -0.8 & 0.8 & 1.1 \\
\hline & 7.0 & 25.5 & 0.0 & 0.06 & 25.1 & 0.20 & 60.4 & 0.66 & -0.2 & -0.4 & 0.4 & 0.6 \\
\hline & 7.0 & 9.5 & 6.8 & 0.06 & 28.9 & 0.17 & 60.1 & 0.59 & -0.2 & -0.6 & 0.1 & 0.6 \\
\hline \multirow{5}{*}{3} & 9.5 & 13.5 & 9.8 & .08 & 14.0 & 0.16 & 60.0 & 0.77 & 0.3 & 0.5 & 0.0 & 0.6 \\
\hline & 9.5 & 17.5 & 9.8 & 0.10 & 17.9 & 0.25 & 60.8 & 0.75 & 0.3 & 0.4 & 0.8 & 0.9 \\
\hline & 9.5 & 21.5 & 9.8 & 0.07 & 21.4 & 0.09 & 60.8 & 0.60 & 0.3 & -0.1 & 0.8 & 0.9 \\
\hline & 9.5 & 25.5 & 9.8 & 0.10 & 25.3 & 0.23 & 60.3 & 0.74 & 0.3 & -0.2 & 0.3 & 0.5 \\
\hline & 9.5 & 29.5 & 9.8 & 0.07 & 28.8 & 0.16 & 59.7 & 0.60 & 0.3 & -0.7 & -0.3 & 0.8 \\
\hline \multirow{5}{*}{6} & 12.0 & 13.5 & 12.0 & 0.11 & 4.4 & 0.09 & 60.3 & 0.60 & 0.0 & 0.9 & 0.3 & 0.9 \\
\hline & 12.0 & 17.5 & 12.0 & .11 & 3 & & 8 & & 0 & 8 & 8 & 1.1 \\
\hline & 12.0 & 5 & 1 & 0 & .0 & 8 & 4 & 58 & 0.1 & .5 & .4 & 0.6 \\
\hline & 12.0 & 25.5 & 11.8 & 0.09 & 26.1 & 0.20 & 60.4 & 0.85 & -0.2 & 0.6 & 0.4 & 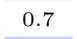 \\
\hline & 12.0 & 29.5 & 11.8 & 0.10 & 29.7 & 0.21 & 60.5 & 0.64 & -0.2 & 0.2 & 0.5 & 0.6 \\
\hline \multirow{5}{*}{7} & 14.5 & 13.5 & 14.2 & 0.13 & 3.8 & 0.10 & 0.4 & 0.74 & -0.3 & .3 & 4 & 0.6 \\
\hline & 14.5 & 17.5 & 14.3 & 0.17 & 17.7 & 0.09 & 61.3 & 0.79 & -0.2 & 0.2 & 1.3 & 1.3 \\
\hline & 14.5 & 21.5 & 14.2 & 0.12 & 21.2 & 0.12 & 60.7 & 0.79 & -0.3 & -0.3 & 0.7 & 0.8 \\
\hline & 14.5 & 25.5 & 14.0 & 0.14 & 25.0 & 0.21 & 59.7 & 0.68 & -0.5 & -0.5 & -0.3 & 0.8 \\
\hline & 14.5 & 29.5 & 14.1 & 0.13 & 28.9 & 0.21 & 60.6 & 0.64 & -0.4 & -0.6 & 0.6 & 0.9 \\
\hline
\end{tabular}

dard deviation of measurements with increased source-detector distance is expected, since increasing this distance results in a smaller number of photons being collected within the fixed acquisition period, reducing the signal to noise ratio in the calculation of the projection's CoM. However, in therapeutic practice, the dwell time of the source is usually much longer than $0.5 \mathrm{~s}$; therefore, this uncertainty will be reduced when the probe is put into clinical use. The accuracy obtained in these experiments closely matched the performance obtained from previously published simulation studies, although the errors are somewhat larger due to source positioning uncertainties which are not present in the simulation ${ }^{30}$.

Several factors contribute to the error in the estimated position in the $z$ direction. Firstly, although the phantom itself is solid, and therefore the channel in which the catheter is inserted is rigid, there is a small difference of $0.333 \mathrm{~mm}$ between the diameter of the channel $(2 \mathrm{~mm})$ and the diameter of the catheter (Flexitron Lumencath $5 \mathrm{~F}, 1.667 \mathrm{~mm}$ ). Due to the position of the afterloader relative to the phantom, a small downward force is exerted on the external part of the catheter, which is greater at (nominal) $z=60 \mathrm{~mm}$ than for $z=40 \mathrm{~mm}$. 


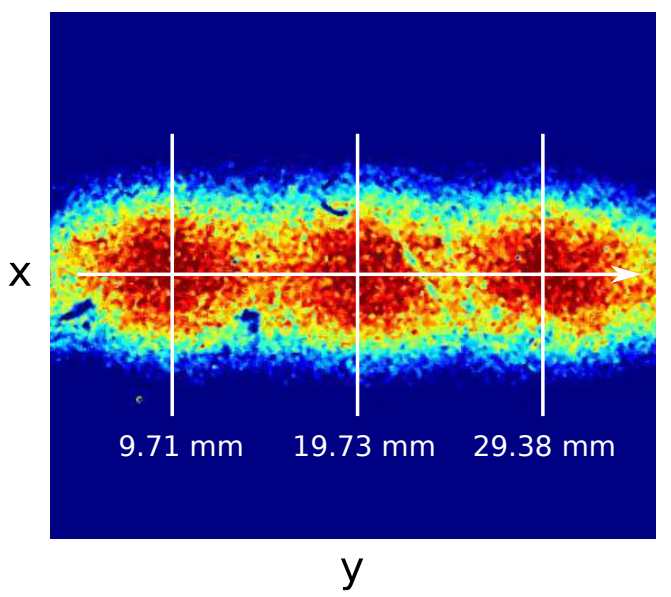

FIG. 6. Magnified film image obtained for three source positions at $z=60 \mathrm{~mm}$, with $y$-axis steps of $10 \mathrm{~mm}$. The image obtained for $z=40 \mathrm{~mm}$ is essentially identical.

TABLE IV. Measured position of source in $y$ direction, as measured by EBT3 film placed $5 \mathrm{~mm}$ from the source and the HDR BrachyView probe. Uncertainties are $\pm 3 \sigma$; source centre displacement measured from edge of phantom.

\begin{tabular}{|c|c|c|c|}
\hline \multirow[b]{2}{*}{ Nominal $z(\mathrm{~mm})$} & \multicolumn{2}{|c|}{ Estimated source centre displacement $(\mathrm{mm})$} & \multirow[b]{2}{*}{ Difference $(\mathrm{mm})$} \\
\hline & Film (mm) & HDR BrachyView (mm) & \\
\hline \multirow{3}{*}{40} & $9.96 \pm 0.78$ & $10.05 \pm 0.24$ & -0.09 \\
\hline & $19.60 \pm 0.78$ & $19.87 \pm 0.36$ & -0.27 \\
\hline & $29.68 \pm 0.78$ & $29.86 \pm 0.27$ & -0.18 \\
\hline \multirow{3}{*}{60} & $9.71 \pm 0.74$ & $9.9 \pm 0.33$ & -0.19 \\
\hline & $19.73 \pm 0.74$ & $19.84 \pm 0.42$ & -0.11 \\
\hline & $29.38 \pm 0.74$ & $29.69 \pm 0.48$ & -0.31 \\
\hline
\end{tabular}

This force results in the catheter being deflected into an approximately parabolic shape inside the channel through the phantom. The central region of the catheter is deflected upward, while the catheter is deflected downward at both the tip of the catheter and at the point of entrance to the phantom. This partially explains the discrepancy between the planned and measured values of $z$ at the nominal height of $z=60 \mathrm{~mm}$ - rather than being a measurement error, it is an error in the accuracy of the experimental configuration, which is being correctly measured by HDR BrachyView.

The remainder of the error (at both $z=60 \mathrm{~mm}$ and $z=40 \mathrm{~mm}$ ) is a consequence 


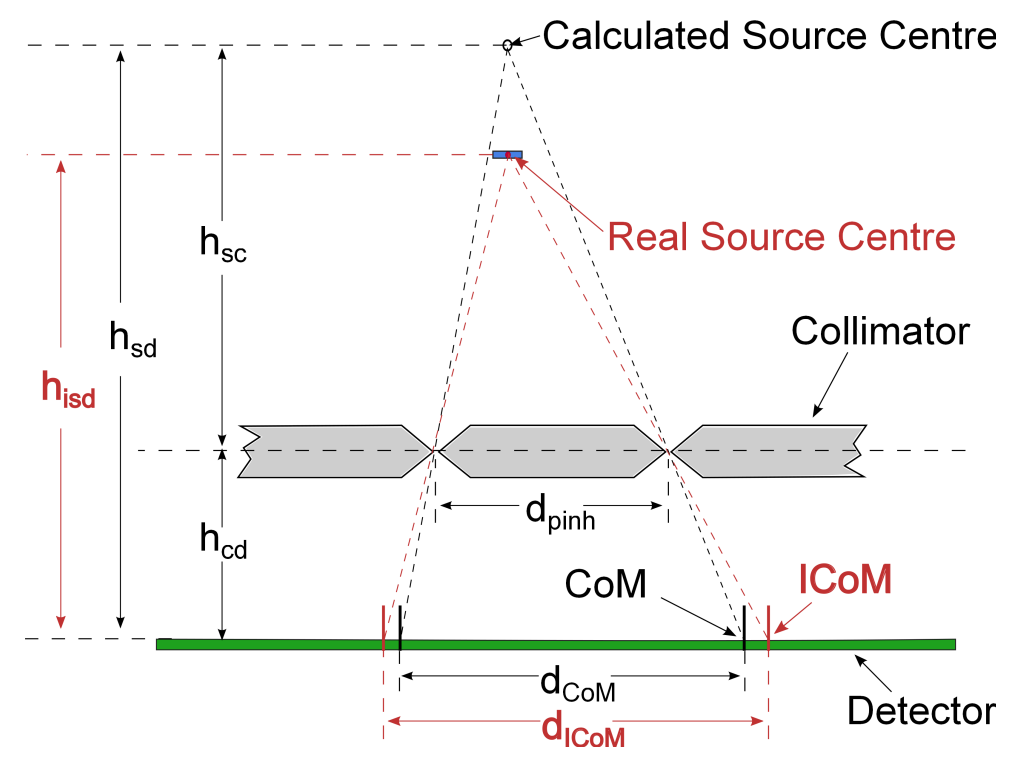

FIG. 7. Illustration of the calculation of $z$, demonstrating the overestimation in $z$ that results from using centres of mass of non-uniform-intensity source projections on the imaging plane. $d_{I C o M}$ and $d_{C o M}$ refer to the distances between adjacent 'ideal' (i.e. the theoretical projection of the true source centre through the pinhole onto the imaging plane) and the computed centres of mass of the image respectively.

of the fact that the centre of mass (CoM) of the projection is not precisely collinear with the source and pinhole, but rather is shifted slightly both in $x$ and $y$ in the direction of the pinhole. The shift is due to the source being cylindrical rather than a point source, with photons from the more distant end of the source travelling a greater distance to the detector plane (and arriving at a shallower angle) compared to photons emitted from the near end. Because of the difference in path distances, the intensity (but not the geometry) of projections of a cylindrical source with uniform activity distribution oriented parallel to the detector plane will be non-uniform. The CoM will therefore be biased in the direction of maximum projection intensity (in the direction of the pinhole) and will not perfectly coincide with the true geometric centre of the projection.

As can be seen from FIG. 7, the error resulting from the use of the projection CoM to estimate the geometric centre of the projection causes an overestimation of the height of the source (i.e., a positive error in $z$ ). This error is smallest for the projections nearest to the source in $x$ and $y$, progressively increasing in the more distant projections. Currently, all projections are equally weighted during the position reconstruction process (with the 
estimated position being calculated as the point with minimum mean squared distance from all backprojected lines). Therefore, position estimates for those source positions from which a larger number of distant projections are visible will be more severely degraded by this error than those from which only a few nearby projections can be seen. This explains the apparently paradoxical result that source positions above the middle of the detector array, from which multiple projections are visible, accumulate a greater positive bias in height estimation compared to source positions near either end of the detector array, which effectively give more weight to the projection with a smaller error between projection CoM and the true geometric centre of the projection. The same error was observed in the previous Monte Carlo simulation study, confirming that it is a consequence of the simplified methods used to estimate the geometric centre of the projection from the projection's $\mathrm{CoM}^{30}$.

This latter source of error will be reduced in future versions of the reconstruction software, by using a non-equal weighting of projections (preferentially weighting the brighter projections, which also gives greater weight to projections with higher SNR). It will also be possible to improve the localisation of the geometric centre of the projection by deconvolving the projection by the point spread function of the pinhole (which will reduce blurring, although it cannot eliminate the effect of photon penetration through the collimator) and iterative post-segmentation intensity correction (i.e., following initial segmentation and approximate determination of the CoMs and hence source position, the intensity of the projection can be corrected for the $1 / d^{2}$ reduction in intensity, after which the CoM should be equal to the geometric centre of the projection).

It is also noted that there is some variation in $y$ between individual runs of the experiment; this is due to the afterloader's own intrinsic positioning error of $\pm 1 \mathrm{~mm}(95 \%$ confidence interval). Incremental movements in $y$ during each individual run remain highly consistent, but due to mechanical backlash in the afterloader's actuator following the complete retraction and reinsertion of the source, combined with up to $0.5 \mathrm{~mm}$ uncertainties in the connection between transfer tube and catheters between runs, small, random inter-run translations in $y$ are observed. This is demonstrated by the fact that intra-run standard deviation of the difference between planned and measured source positions is always smaller than the overall uncertainties of the same values (inter-run standard deviations).

The intrinsic accuracy of HDR BrachyView (i.e., independent of afterloader positioning error) in the $y$ direction was further evaluated using Gafchromic films. Excellent agree- 
ment is observed from the results of film and HDR BrachyView probe. The difference in $y$ displacement between the measurements by film and the HDR BrachyView probe is significantly smaller than the error between the estimated and planned source positions. This is further confirmation that the catheter shift and uncertainty in its connection to the transfer tube or afterloader positioning uncertainty are the main reason for the differences between planned and estimated source position in the $y$ direction shown in Tables II and III.

\section{CONCLUSION}

In this paper, the results of the experimental evaluation of the HDR BrachyView probe in terms of source tracking accuracy have been presented. Most planned source positions (more than $90 \%$ ) were measured with sub-millimetre accuracy within an equivalent acquisition time of $0.5 \mathrm{~s}$ for a full strength HDR source, with the largest difference found between planned and measured source position being $1.3 \mathrm{~mm}$ (although part of that error is a result of uncertainties in the mechanical positioning of the source). The overall performance, including a systemic overestimation of the source height, was in good agreement with the results of the previous GEANT4 simulation study, and can potentially be improved through more sophisticated image processing techniques. In future work, a fully-populated four-detector prototype with integrated transrectal ultrasound is under development, which will be able to register the calculated source position with the prostate volume.

\section{ACKNOWLEDGMENTS}

The authors would like to thank the National Health and Medical Research Council for its support of the research and development of the BrachyView probe under the NHMRC Research Grant No. 573428. The authors would also like to acknowledge the support provided by the MEDIPIX collaboration.

* Email: anatoly@uow.edu.au; A.B. Rosenfeld 
1 Y. Wang, R. Sankreacha, A. Al-Hebshi, A. Loblaw and G. Morton. Comparative study of dosimetry between high-dose-rate and permanent prostate implant brachytherapies in patients with prostate adenocarcinoma. Brachytherapy, 5(4):251--255, 2006.

2 G. Morton and P. Hoskin. Brachytherapy: Current Status and Future Strategies-Can High Dose Rate Replace Low Dose Rate and External Beam Radiotherapy? Clin. Oncol., 25(8):474-482, 2013.

${ }^{3}$ G. Morton. High-dose-rate brachytherapy boost for prostate cancer: rationale and technique. J. Contemp. Brachytherapy, 6(3):323-330, 2014.

4 A. Challapalli, E. Jones, C. Harvey, G. Hellawell, and S. Mangar. High dose rate prostate brachytherapy: an overview of the rationale, experience and emerging applications in the treatment of prostate cancer. Br. J. Radiol., 85 Spec No 1:S18-S27, 2012.

5 S. Gao, M.E. Delclos, L.C. Tomas, C.H. Crane and S. Beddar. High-dose-rate remote afterloaders for intraoperative radiation therapy. AORN J, 86(5):827-836, Nov 2007.

6 N. Kovalchuk, K. Furutani, K. MacDonald, and T. Pisansky. Dosimetric effect of interfractional needle displacement in prostate high-dose-rate brachytherapy. Brachytherapy, 11(2):111-118, 2012 .

7 K. Tanderup, S. Beddar, C.E. Andersen, G. Kertzscher, and J.E. Cygler. In vivo dosimetry in brachytherapy. Med. Phys., 40(7):070902, Jul 2013.

8 G. Kertzscher, A. Rosenfeld, S. Beddar, K. Tanderup, and J.E. Cygler. In vivo dosimetry: trends and prospects for brachytherapy. Brit. J. Radiol., 87(1041):20140206, Sep 2014.

9 J. Lambert, T. Nakano, S. Law, J. Elsey, D. R. McKenzie, and N. Suchowerska. In vivo dosimeters for HDR brachytherapy: a comparison of a diamond detector, MOSFET, TLD, and scintillation detector. Med. Phys., 34(5):1759-1765, 2007.

10 Z. Qi, X. Deng, S. Huang, J. Lu, M. Lerch, D. Cutajar, and A. Rosenfeld. Verification of the plan dosimetry for high dose rate brachytherapy using metal-oxide-semiconductor field effect transistor detectors. Med. Phys., 34(6):2007-2013, 2007.

11 E.L. Seymour, S.J. Downes, G.B. Fogarty, M.A. Izard, and P. Metcalfe. In vivo real-time dosimetric verification in high dose rate prostate brachytherapy. Med. Phys., 38(8):4785-4794, 2011.

12 I.S. Kwan, A.B. Rosenfeld, Z.Y. Qi, D.Wilkinson, M.L.F. Lerch, D.L. Cutajar, M. Safavi-Naeni, M. Butson, J.A. Bucci, Y. Chin, and V.L. Perevertaylo. Skin dosimetry with new MOSFET 
13 I. Kwan, M. Lerch, A. Rosenfeld, A. Howie, Y. Chin, J. Bucci, B. Lee, and V. Perevertaylo. Measurement of rectal dose during HDR brachytherapy using the new MOSkin dosimeter. $J$. Nucl. Sci. Technol., 45-5S:481-484, 2008.

14 C. Tenconi, M. Carrara, M. Borroni, A. Cerrotta, D. Cutajar, M. Petasecca, M. Lerch, J. Bucci,

detectors. Radiat. Meas., 43:929-932, 2008.

G. Gambarini, E. Pignoli, and A. Rosenfeld. TRUS-probe integrated MOSkin detectors for rectal wall in vivo dosimetry in HDR brachytherapy: in phantom feasibility study. Radiat. Meas., 71:379-383, 2014.

15 M. Carrara, C. Tenconi, M. Borroni, A. Cerrotta, C. Fallai, D. Cutajar, M. Petasecca, J. Bucci, G. Gambarini, A. Rosenfeld, and E. Pignoli. MOSkin Detectors for On Line Dosimetry in HDR Ultrasound-Guided Prostate Brachytherapy: Rectal Wall (In Vivo) and Urethra (In Phantom) Measurements. Brachytherapy, 13, Supplement 1(0):S54-S55, 2014.

16 N. Suchowerska, J. Lambert, T. Nakano, S. Law, J. Elsey, and D.R. McKenzie. A fibre optic dosimeter customised for brachytherapy. Radiat. Meas., 42:929-932, 2007.

17 G. Kertzscher, C. Andersen, J. Edmund, and K. Tanderup. Stem signal suppression in fibercoupled $\mathrm{Al}_{2} \mathrm{O}_{3}: \mathrm{C}$ dosimetry for ${ }^{192}$ Ir brachytherapy. Radiat. Meas., 46(12):2020-2024, 2011.

18 G. Kertzscher, C. Andersen, F. Siebert, S. Nielsen, J. Lindegaard, and K. Tanderup. Identifying afterloading PDR and HDR brachytherapy errors using real-time fiber-coupled $\mathrm{Al}_{2} \mathrm{O}_{3}: \mathrm{C}$ dosimetry and a novel statistical error decision criterion. Radiother. Oncol., 100(3):456-462, 2011.

19 L.W. Cartwright, N. Suchowerska, Y. Yin, J. Lambert, M. Haque, and D.R. McKenzie. Dose mapping of the rectal wall during brachytherapy with an array of scintillation dosimeters. Med. Phys., 37(5):2247-2255, 2010.

20 R. Wang, J. Ribouton, P. Pittet, P. Guiral, P. Jalade, and G. Lu. Implementation of GaN based real-time source position monitoring in HDR brachytherapy. Radiat. Meas., 71:293-296, 2014.

21 A. Espinoza, B. Beeksma, M. Petasecca, I. Fuduli, C. Porumb, D. Cutajar, S. Corde, M. Jackson, M. Lerch, and A. Rosenfeld. The feasibility study and characterization of a two-dimensional diode array in "magic phantom" for high dose rate brachytherapy quality assurance. Med. Phys., 40(11):111702, 2013.

22 A. Espinoza, M. Petasecca, I. Fuduli, A. Howie, J. Bucci, S. Corde, M Jackson, M.L. Lerch, and A.B. Rosenfeld. The evaluation of a 2D diode array in "magic phantom" for use in high 
dose rate brachytherapy pretreatment quality assurance. Med. Phys., 42(2):663-673, February 2015.

23 A. Tiong, S. Bydder, M. Ebert, N. Caswell, D. Waterhouse, N. Spry, P. Camille, and D. Joseph. A small tolerance for catheter displacement in high-dose rate prostate brachytherapy is necessary and feasible. Int. J. Radiat. Oncol., 76(4):1066-1072, 2010.

24 M. Batic, J. Burger, V. Cindro, G. Kramberger, I. Mandic, M. Mikuz, A. Studen, and M. Zavrtanik. Verification of High Dose Rate Ir Source Position During Brachytherapy Treatment Using Silicon Pixel Detectors. IEEE T. Nucl. Sci., 58(5):2250-2256, 2011.

25 J. Duan, D.J. Macey, P.N. Pareek, and I. A. Brezovich. Real-time monitoring and verification of in vivo high dose rate brachytherapy using a pinhole camera. Med. Phys., 28(2):167-173, 2001.

26 H. Song, J. Bowsher, S. Das, and F. Yin. Tracking brachytherapy sources using emission imaging with one flat panel detector. Med. Phys., 36(4):1109-1111, 2009.

27 X. Llopart, R. Ballabriga, M. Campbell, L. Tlustos, and W. Wong. Timepix, a 65k programmable pixel readout chip for arrival time, energy and/or photon counting measurements. Nucl. Instrum. Meth. A, 581(1-2):485-494, 2007.

28 M. Petasecca, K Loo, M. Safavi-Naeini, Z. Han, P. Metcalfe, S. Meikle, S. Pospisil, J. Jakubek, J. Bucci, M. Zaider, M. Lerch, Y. Qi, and A. Rosenfeld. Brachyview: Proof-of-principle of a novel in-body gamma camera for low dose-rate prostate brachytherapy. Med. Phys., 40(4):041709, 2013.

29 K.J. Loo, J. Jakubek, J. Zemlicka, M. Petasecca, M. Safavi-Naeini, J. Bucci, M. Zaider, and A.B. Rosenfeld. BrachyView: Feasibility study into the application of Timepix detectors for soft tissue thickness imaging in prostate brachytherapy treatment. Radiat. Meas., 71:329-332, 2014 .

30 M. Safavi-Naeini, Z. Han, D. Cutajar, S. Guatelli, M. Petasecca, M.L.F. Lerch, D.R. Franklin, J. Bucci, M. Zaider, and A.B. Rosenfeld. BrachyView, a novel inbody imaging system for HDR prostate brachytherapy: design and Monte Carlo feasibility study. Med. Phys., 40(7):071715, 2013.

31 Z. Han, M. Safavi, S. Alnaghy, D. Cutajar, S. Guatelli, M. Petasecca, M. Lerch, D. Franklin, A. Malaroda, M. Carrara, J. Bucci, M. Zaider, and A. Rosenfeld. Radiation dose enhancement at tissue-tungsten interfaces in HDR brachytherapy. Phys. Med. Biol., 59(21):6659, 2014. 